3-1-1987

\title{
Multicritical Susceptibility Sum Rules
}

\author{
Miron Kaufman \\ Cleveland State University, m.kaufman@csuohio.edu \\ Michael Ma \\ University of Cincinnati - Main Campus
}

Follow this and additional works at: https://engagedscholarship.csuohio.edu/sciphysics_facpub

Part of the Physics Commons

How does access to this work benefit you? Let us know!

Publisher's Statement

Copyright 1987 American Physical Society. Available on publisher's site at http://pra.aps.org/ abstract/PRA/v35/i5/p2369_1.

\section{Original Citation}

Kaufman, Miron and Michael Ma. "Multicritical Susceptibility Sum Rules." Physical Review A 35 (1987): 2369-2372.

\section{Repository Citation}

Kaufman, Miron and Ma, Michael, "Multicritical Susceptibility Sum Rules" (1987). Physics Faculty Publications. 135. https://engagedscholarship.csuohio.edu/sciphysics_facpub/135

This Article is brought to you for free and open access by the Physics Department at EngagedScholarship@CSU. It has been accepted for inclusion in Physics Faculty Publications by an authorized administrator of EngagedScholarship@CSU. For more information, please contact library.es@csuohio.edu. 


\title{
Multicritical susceptibility sum rules
}

\author{
Miron Kaufman \\ Department of Physics, Cleveland State University, Cleveland, Ohio 44115 \\ Michael Ma \\ Department of Physics, University of Cincinnati, Cincinnati, Ohio 45221
}

(Received 8 September 1986)

\begin{abstract}
Asymptotically close to the $N$ th-order multicritical point of an $N$-phase system, there are $N-1$ sum rules involving the mean-field susceptibilities measured in each of the coexisting phases. These sum rules provide the experimentalist with a convenient and stringent test of the theory. In particular, they facilitate the detection of nonclassical effects, especially valuable for $N>3$ where the fluctuation effect is dominated by the classical contribution for three-dimensional systems.
\end{abstract}

The theory of multicritical points is, in general, prone to ambiguous experimental verifications because of the large number of relevant thermodynamic fields compared to a simple critical point. This is particularly true for liquid mixtures, where symmetry-breaking fields have to be considered because of the lack of symmetries otherwise present in magnets. In experiments, densities rather than chemical potentials are kept fixed, which further complicates the power-law analyses of singular quantities. It is therefore desirable to have theoretical predictions involving measurable quantities; predictions not strongly dependent on the precise thermodynamic state of the system or on the path of approaching the multicritical point. Accordingly, Kaufman, Bardhan, and Griffiths ${ }^{1}$ have derived from Griffiths's classical theory ${ }^{2}$ of tricritical points a sum rule involving susceptibilities measured in each phase of a three-phase near-tricritical system.

In this paper we show that a similar sum rule holds for any $N$-phase system close to an $N$ th-order multicritical point. We also show, more generally, that there are $N-1$ sum rules involving not only the order-parameter susceptibility but less divergent susceptibilities as well. Hence for a tricritical point $N=3$, there is a second sum rule involving a nonordering susceptibility. This sum rule is different from the second sum rule of Ref. 1 .

Experimental studies ${ }^{3,4}$ of the first tricritical sum rule revealed deviations from the asymptotic prediction. In a detailed thermodynamic model ${ }^{5}$ of the ammonium sulfate + water + ethanol + benzene mixture based on the composition data of Lang and Widom, ${ }^{6}$ deviations from the first tricritical sum rule, comparable to the experimental ones, ${ }^{3}$ were produced by the factors of proportionality connecting the intensity of scattered light and the square of the correlation length, respectively, to the orderparameter susceptibility. Deviations from the sum rules are also induced ${ }^{7}$ by the nonclassical spatial fluctuations of the order parameter. This provides further motivation for studying the sum rules: Since they are combinations of diverging quantities (combinations which vanish at the mean-field level), corrections to scaling including nonclassical ones become the leading contributions, thus facilitating their experimental detection. ${ }^{4}$

We now derive the sum rules from the classical theory of an Nth-order multicritical point. The singular part of the thermodynamic potential ${ }^{5}$ is

$$
\Omega_{s}=\min _{\psi} \phi(\psi),
$$

where $\phi(\psi)=\sum_{n=1}^{2 N} a_{n} \psi^{n}, a_{2 N}>0$, and $\psi$ is the order parameter. At the multicritical point $\psi=a_{1}=a_{2}=\ldots$ $=a_{2 N-1}=0$. When $N$ phases coexist

$$
\phi(\psi)=a_{2 N}\left(\prod_{n=1}^{N}\left(\psi-\psi_{n}\right)^{2}-\prod_{n=1}^{N} \psi_{n}^{2}\right) .
$$

The singular contributions to the susceptibilities are ${ }^{5}$

$$
\chi_{i j}=-\frac{\partial^{2} \Omega_{s}}{\partial a_{i} \partial a_{j}}=i j \psi^{i+j-2}\left(\frac{d^{2} \phi}{d \psi^{2}}\right)^{-1} .
$$

In the $n$th phase $\psi=\psi_{n}$ and

$$
\chi_{i j}^{(n)}=i j \psi_{n}^{i+j-2}\left(2 a_{2 N} \prod_{\substack{m=1 \\(m \neq n)}}^{N}\left(\psi_{n}-\psi_{m}\right)^{2}\right)^{-1} .
$$

We derive now a set of $N-1$ identities concerning any $N$ numbers. We start by defining an $N$ th order polynomial:

$$
P(\psi)=\left(\psi-\psi_{1}\right)\left(\psi-\psi_{2}\right) \ldots\left(\psi-\psi_{N}\right) .
$$

Next we integrate $\psi^{k-1}[P(\psi)]^{-1}$ on a circle of large radius $R$ centered on the origin of the complex plane:

$$
\oint_{d \psi \psi^{k-1}[P(\psi)]^{-1} \propto R^{k-N} \rightarrow 0},
$$

for $R \rightarrow \infty$ if $k=1,2, \ldots, N-1$. By expressing the line integral as the sum of residuals of the integrand we find

$$
\sum_{n=1}^{N} \frac{\psi_{n}^{k-1}}{\prod_{\substack{m=1 \\ m \neq n}}^{N}\left(\psi_{n}-\psi_{m}\right)}=0 \text { for } k=1,2, \ldots, N-1 .
$$

The $n$th term in this sum is, up to the sign, proportional to $\left(\chi_{i j}^{(n)}\right)^{1 / 2}$ [Eq. (4)] if $i+j=2 k$. Without loss of generality we assume $\psi_{1}<\psi_{2}<\ldots<\psi_{n}$ and then rewrite Eq. (7) 
as the susceptibilities sum rules

$$
\Sigma_{N, k} \equiv \sum_{n=1}^{N}(-1)^{n}\left(\operatorname{sgn} \psi_{n}\right)^{k-1}\left(\chi_{i j}^{(n)}\right)^{1 / 2}=0,
$$

which is equivalent to

$$
R_{N, k} \equiv\left(\sum_{n=1,3, \ldots,}\left(\operatorname{sgn} \psi_{n}\right)^{k-1}\left(\chi_{i j}^{(n)}\right)^{1 / 2}\right) /\left(\sum_{n=2,4, \ldots,}\left(\operatorname{sgn} \psi_{n}\right)^{k-1}\left(\chi_{i j}^{(n)}\right)^{1 / 2}\right)=1
$$

where $\operatorname{sgn} \psi_{n}$ is +1 if $\psi_{n}>0$ and -1 if $\psi_{n}<0$, and $k=1,2, \ldots, N-1$. We order the sum rules according to the values of $k$, i.e., the first sum rule for $k=1$, the second sum rule for $k=2$, etc. The only sum rule published ${ }^{1}$ previously is the first tricritical sum rule, $N=3, k=1$.

To account for corrections to scaling within the classical theory, we keep in $\phi(\psi)$ powers of $\psi$ higher than $2 N$ :

$\phi(\psi)=v(\psi) a_{2 N}\left(\prod_{n=1}^{N}\left(\psi-\psi_{n}\right)^{2}\right)-a_{2 N} \prod_{n=1}^{N} \psi_{n}^{2}$,

where $v(\psi)$ is a positive polynomial, $v(\psi)>0$, and $v(0)=1$. Then from Eqs. (3) and (10) we find

$\chi_{i j}^{(n)}=i j \psi_{n}^{i+j-2}\left(2 a_{2 N} v\left(\psi_{n}\right) \underset{\substack{m=1 \\(m \neq n)}}{N}\left(\psi_{n}-\psi_{m}\right)^{2}\right)^{-1}$.

By expanding $\left[v\left(\psi_{n}\right)\right]^{-1 / 2}$ and using the identities of Eq. (7), we find that, to leading order, $\Sigma_{N, k}$, defined in Eq. (8) is a constant:

$$
\Sigma_{N, k}=\text { const . }
$$

The coefficient $a_{3 N-k}$ of $\psi^{3 N-k}$ in the power expansion of $\phi(\psi)$ contributes to the constant. Hence, to obtain the leading nonvanishing classical correction to the asymptotic sum rules, all terms up to $\psi^{3 N-k}$ have to be kept in $\phi(\psi)$, e.g., for the first tricritical sum this is ${ }^{4} \psi^{8}$.

An equivalent effect is obtained by allowing the proportionality factor relating a measured quantity to a theoretical susceptibility to depend on $\psi$ [e.g., scattered light intensity $I \cong(d \varepsilon / d \psi)^{2} \chi_{11}$, where $\varepsilon(\psi)$ is the dielectric constant]. The leading nonvanishing correction to $\Sigma_{N, k}$ expressed now in terms of the experimental quantity, e.g., intensity $I$, is again a constant. It is obtained by keeping in the expansion of the proportionality factor, e.g., $(d \varepsilon / d \psi)^{2}$, all powers up to $\psi^{N-k}$. For the first tricritical sum rules this is ${ }^{4,5} \psi^{2}$.

Spatial fluctuations of the order parameter induce nonclassical deviations from the sum rules. After adding the squared gradient of $\psi, \frac{1}{2}(\nabla \psi)^{2}$ to the classical $\phi(\psi)$ it can be shown, to first order in the loop expansion, that the deviation of any thermodynamic quantity $C$ from its meanfield value $C_{\mathrm{MF}}$ is

$$
C=C_{\mathrm{MF}}\left[1+O\left(\left|a_{2 N-2}\right|^{(N-1)\left(d-d_{c}\right) / 2}\right)\right],
$$

where $d$ is the spatial dimension and $d_{c}=2 N /(N-1)$ is the upper critical dimension. The field $a_{2 N-2}$ measures the distance to the $N$ th order multicritical point. It is proportional to the reduced temperature for ammonium sulfate + water + ethanol + benzene $e^{3,5,6}$ and to the departure of the carbon number from its tricritical value for the quasibinary mixtures of Ref. 4. By using Eq. (12) and the classical result $\chi_{i j} \propto\left|a_{2 N-2}\right|^{k-N}$, where $i+j=2 k$, we find the leading nonclassical correction to the sum rules:

$$
\Sigma_{N, k}=O\left(\left|a_{2 N-2}\right|^{(N-1)(d-3) / 2+(k-3) / 2}\right),
$$

or

$$
R_{N, k}=1+O\left(\left|a_{2 N-2}\right|^{(N-1)\left(d-d_{c}\right) / 2}\right) .
$$

In three dimensions

$$
\Sigma_{N, k}=0\left(\left|a_{2 N-2}\right|^{(k-3) / 2}\right),
$$

i.e., the exponent is $(k-3) / 2$ independent of $N$, and

$$
R_{N, k}=1+O\left(\left|a_{2 N-2}\right|^{(N-3) / 2}\right) .
$$

It follows that the nonclassical correction is stronger than the classical correction $\left[\Sigma_{N, k} \propto\left|a_{2 N-2}\right|^{0}\right.$, see Eq. (12)] for $k<3$, while the reverse is true for $k>3$. For the threedimensional tricritical point, $d_{c}=3$, logarithmic deviations from the sum rules are produced by the marginal field $a_{6} \propto|\ln | a_{4}||^{-1}$ as shown by Rudnick and Jasnow ${ }^{7}$ for the first sum rule:

$$
R_{3,1}=1+O\left(|\ln | a_{4}||^{-1 / 2}\right) .
$$

We now offer further remarks on the sum rules for the three-dimensional critical, tricritical, and fourth-order multicritical points. If $N=2$, i.e., critical point, there is a single sum rule which reduces to $\chi_{11}^{(1)} / \chi_{11}^{(2)}=1$. This classical prediction survives the divergent fluctuations $\left(d=3<4=d_{c}\right)$ because the only relevant symmetrybreaking field is zero on the coexistence manifold close to the critical point.

For $N=3$ we have two tricritical sum rules

$$
\left(\chi_{11}^{(1)}\right)^{1 / 2}+\left(\chi_{11}^{(3)}\right)^{1 / 2}=\left(\chi_{11}^{(2)}\right)^{1 / 2} \text {, }
$$

and

$\left|\left(\chi_{i j}^{(1)}\right)^{1 / 2}-\left(\chi_{i j}^{(3)}\right)^{1 / 2}\right|=\left(\chi_{i j}^{(2)}\right)^{1 / 2}$ for $i+j=2 k=4$.

Equation (17) is obtained from Eq. (8), $N=3, k=2$, by assuming $\psi_{1}<0$ and $\psi_{3}>0$. These signs for $\psi_{1}$ and $\psi_{3}$ follow from the convention $\psi_{1}<\psi_{2}<\psi_{3}$ and from the choice $^{1,2,5}$ of the origin of $\psi$ such that $\psi_{1}+\psi_{2}+\psi_{3}=0$. Observed $^{3,4}$ deviations of roughly $20 \%$ from the first tricritical sum rule could be a result of (i) the order parameter dependence ${ }^{1,5}$ of the coefficient relating the measured intensity of scattered light to the susceptibility, or (ii) the spatial fluctuations ${ }^{7}$ of the order parameter. These fluctuations induce deviations ${ }^{8,9}$ from the mean-field predictions for other ratios such as $\left(x_{U^{\prime}}-x_{L^{\prime}}\right) /\left(x_{L_{c}}-x_{U_{c}}\right)$, denoted $R_{1 / c}$ in Ref. 10, where $x_{U_{c}}, x_{U^{\prime}}, x_{L_{c}}$, and $x_{L^{\prime}}$ are composi- 
tions of the critical phase and the coexisting noncritical phase at the two critical end points $U$ and $L$ at a given distance away from the tricritical point. In fact, a $20 \%$ fluctuation deviation from the susceptibility sum rule corresponds $^{11}$ to about $10 \%$ fluctuation deviation for $R_{1 / c}$. However, a deviation of only 5\% (within experimental errors) from the classical value of $R_{1 / c}$ is observed in quasibinary mixtures, ${ }^{10}$ and this ratio takes the classical value of 2 (within experimental errors) close to the tricritical temperatures of two quaternary mixtures. ${ }^{12}$ Furthermore, various ratios of intensities and correlation lengths $\xi$, which should be equal if $I$ and $\xi^{2}$ are strictly proportional to $\chi_{11}$, differ systematically. ${ }^{5,13}$ This evidence suggests that the observed departures from the first tricritical sum rule are due in part to the fact that $I / \chi_{11}$ and $\xi^{2} / \chi_{11}$, respectively, take different values in the different coexisting phases. ${ }^{1,5}$ In the immediate vicinity of the tricritical point, however, the fluctuation correction dominates the classical one because $k=1<3$.

The nonordering susceptibility $\chi_{22}$ was inferred ${ }^{8}$ from various measurements on the symmetrical tricritical systems: dyprosium aluminium garnet and ${ }^{3} \mathrm{He}-{ }^{4} \mathrm{He}$ mixtures. In the symmetric case $\chi_{22}^{(1)}=\chi_{22}^{(3)}$ and the second rule reduces to $\chi_{22}^{(2)}=0$, i.e., the nonordering susceptibility in the disordered phase is zero. However, $\chi_{22}^{(2)}$ is observed experimentally to diverge. This deviation from the classical prediction is due to the order-parameter fluctuations. Indeed the one-loop correction to the renormalized meanfield theory ${ }^{9}$ gives $^{11}$

$$
\chi_{22}^{(2)} / \chi_{22}^{(1)}=\sqrt{2 a_{6}} / 4 \pi,
$$

where $a_{6} \propto|\ln | a_{4}||^{-1}$. This ratio was calculated within the spherical model by Fisher and Sarbach. ${ }^{8}$ We do not know how to directly measure $\chi_{22}$ in liquid mixtures. However, the classical theory predicts $\chi_{22} \propto \psi^{2} \chi_{11}$, Eq. (3). $\psi$ is a measure of the deviation of any density from its tricritical value, e.g., $n-n_{t} \propto \psi$, where $n$ is the index of refraction and $n_{t}$ is its tricritical value. Therefore, by combining data from a thermodynamic or optical measurement of a composition or index of refraction, and from a light scattering measurement of the leading susceptibility $\chi_{11}$, one can obtain a measure of $\chi_{22}$ and check the second tricritical sum rule of Eq. (17).

For $N=4$, fourth-order multricritical point, the following sum rules hold:

$$
\begin{aligned}
& \left(\chi_{i j}^{(1)}\right)^{1 / 2}-\left(\chi_{i j}^{(2)}\right)^{1 / 2}+\left(\chi_{i j}^{(3)}\right)^{1 / 2}-\left(\chi_{i j}^{(4)}\right)^{1 / 2}=0 \text { for } i+j=2 k=2 \text { or } 6, \\
& \left(\chi_{i j}^{(1)}\right)^{1 / 2}+\left(\chi_{i j}^{(4)}\right)^{1 / 2}=\left|\left(\chi_{i j}^{(2)}\right)^{1 / 2}-\operatorname{sgn}\left(\psi_{2} \psi_{3}\right)\left(\chi_{i j}^{(3)}\right)^{1 / 2}\right| \text { for } i+j=2 k=4 .
\end{aligned}
$$

Equation (20) was obtained from Eq. (8) and from $\psi_{1}<0$ and $\psi_{4}>0$. The signs of $\psi_{1}$ and $\psi_{4}$ follow from the convention $\psi_{1}<\psi_{2}<\psi_{3}<\psi_{4}$ and from the choice of the origin of $\psi$ such that $\psi_{1}+\psi_{2}+\psi_{3}+\psi_{4}=0$. In the symmetric case $\chi_{i j}^{(1)}=\chi_{i j}^{(4)}$ and $\chi_{i j}^{(2)}=\chi_{i j}^{(3)}$ and the first and third sum rules, $k=1$ and 3 , are trivially satisfied. However, for nonsymmetric liquid mixtures these sum rules are not obvious or trivial results. In the symmetric case the second sum rule, Eq. (20), reduces to $\chi_{i j}^{(1)}=\chi_{i j}^{(2)}=\chi_{i j}^{(3)}=\chi_{i j}^{(4)}$, $i+j=4$.

In conclusion, we have derived $N-1$ sum rules valid asymptotically close to an $N$ th order multicritical point. All these classical results hold in three dimensions even for the critical $\left(d_{c}=4\right)$ and the tricritical $\left(d_{c}=3\right)$ point. The nonclassical deviations from the tricritical sum rules vanish, however, logarithmically slowly. Further experimental studies of the sum rules are needed. The first sum rule can be verified in other near-tricritical systems: water + nonionic amphiphile + oil $^{14}$ or polymer mixtures such as polystyrene (molecular weight $1.73 \times 10^{4}$ ) + polystyrene (molecular weight $7.19 \times 10^{5}$ ) + methylcyclohexane. ${ }^{15}$ It can also be analyzed by means of neutron scattering in the metamagnet dyprosium aluminum garnet. A study of the first sum rule in four-phase liquid mixtures close to a fourth-order point constitutes a convenient way to detect nonclassical corrections to scaling. We predict

$$
\Sigma_{4,1} \propto\left|a_{6}\right|^{-1} \text { or } R_{4,1}=1+O\left(\left|a_{6}\right|^{1 / 2}\right),
$$

where $a_{6}$ measures the distance to the multicritical point, and it can be proportional to the reduced temperature. A system suitable for such a study could be sodium dodecylsulfate + water + pentanol + dodecane for which four opalescent coexisting phases have been reported. ${ }^{16}$ Trutzer has independently proved the identities in Eq. (7). ${ }^{17}$

We have benefited from discussions with Victor Trutzer, Robert Griffiths, and David Jasnow. One of us (M.K.) acknowledges the support of the Ohio Board of Regents through a Research Challenge Grant. The other (M.M.) acknowledges the support of the Faculty Research Participation Program administered by the Argonne Division of Education Programs.
${ }^{1}$ M. Kaufman, K. K. Bardhan, and R. B. Griffiths, Phys. Rev. Lett. 44, 77 (1980).

${ }^{2}$ R. B. Griffiths, J. Chem. Phys. 60, 196 (1974).

${ }^{3}$ M. W. Kim, W. I. Goldburg, P. Esfandiari, J. M. H. Levelt Sengers, and E. -S. Wu, Phys. Rev. Lett. 44, 80 (1980); M. W. Kim, W. I. Goldburg, P. Esfandiari, and J. M. H. Levelt Sengers, J. Chem. Phys. 71, 4888 (1979).
${ }^{4}$ A. Kumar, R. Chamberlin, D. S. Cannell, I. L. Pegg, C. M. Knobler, and R. L. Scott, Phys. Rev. Lett. 54, 2234 (1985).

${ }^{5}$ M. Kaufman and R. B. Griffiths, J. Chem. Phys. 76, 1508 (1982).

${ }^{6}$ J. C. Lang, Jr. and B. Widom, Physica A 81, 190 (1975).

7 J. Rudnick and D. Jasnow, Phys. Rev. B 32, 6087 (1985).

${ }^{8}$ M. E. Fisher and S. Sarbach, Phys. Rev. Lett. 41, 1127 (1978). 
${ }^{9}$ M. J. Stephen, J. Phys. C 13, L83 (1980); Phys. Rev. B 12, 1015 (1975).

${ }^{10}$ R. Lindh, I. L. Pegg, C. M. Knobler, and R. L. Scott, Phys. Rev. Lett. 52, 839 (1984).

${ }^{11}$ M. Kaufman (unpublished).

${ }^{12}$ M. Kaufman and R. B. Griffiths, Phys. Rev. Lett. 53, 741 (1984).
${ }^{13}$ I. L. Pegg, M. C. Goh, R. L. Scott, and C. M. Knobler, Phys. Rev. Lett. 55, 2320 (1985).

${ }^{14}$ M. Kahlweit, R. Strey, and P. Firman, J. Phys. Chem. 90, 671 (1986).

${ }^{15}$ M. Nakata and T. Dobashi, J. Chem. Phys. 84, 5782 (1986).

${ }^{16}$ D. Roux and A. M. Belock, Phys. Rev. Lett. 52, 1895 (1984).

${ }^{17} \mathrm{~V}$. Trutzer (private communication). 